\title{
ELECTRONIC STRUCTURE OF DOPED La-Mn-O PEROVSKITES
}

\author{
M. Demeter ${ }^{a, *}$, M. NeumanN $^{a}$, V.R. Galakhov ${ }^{b}$, N.A. OveChKina $^{b}$, \\ E.Z. KURMAEV ${ }^{b}$ AND N.I. LABACHEVSKAYA ${ }^{c}$ \\ ${ }^{a}$ University of Osnabrück, Faculty of Physics \\ Barbarastr. 7, 49069 Osnabrück, Germany \\ ${ }^{b}$ Institute of Metal Physics, Russian Academy of Sciences - Ural Division \\ 620219 Yekaterinburg GSP-170, Russia \\ 'Institute of Chemistry of Solids, Russian Academy of Sciences - Ural Division \\ 620219 Yekaterinburg GSP -145, Russia
}

The electronic structure of the doped perovskite manganese oxides $\mathrm{La}_{1-x} \mathrm{Sr}_{x} \mathrm{MnO}_{3}$ and $\mathrm{La}_{1-x} \mathrm{Ba}_{x} \mathrm{MnO}_{3}$ has been investigated by $\mathrm{X}$-ray photoelectron and X-ray emission spectroscopy. By comparing the $\mathrm{O} K_{\alpha}$ and Mn $L_{\alpha}$ X-ray emission spectra with the valence band X-ray photoelectron spectra we could localize the Mn $3 d$ and $\mathrm{O} 2 p$ states into the valence band. Our data are compared with previous band structure calculations. The value of the Mn $3 s$ splitting does not change with Sr-doping in the concentration region from $x=0.0$ till $x=0.3$. This shows that the doping holes have mainly oxygen $p$ character. The large value of the splitting indicates that the $3 d$ electrons are in the high-spin state.

PACS numbers: $71.70 . \mathrm{Gm}, 79.70 .+\mathrm{q}$

\section{Introduction}

Nowadays great interest exists in manganese perovskite oxides due to the discovery of the colossal magnetoresistance (CMR) effect [1]. The parent compound $\mathrm{LaMnO}_{3}$ is an A-type antiferromagnetic (AFM) $\left(T_{\mathrm{N}}=140 \mathrm{~K}\right)$ insulator with a distorted perovskite structure. The manganese ions are $\mathrm{Mn}^{3+}\left(3 d^{4}\right)$ having three electrons in the $t_{2 \mathrm{~g}}$ band and one in the $e_{\mathrm{g}}$ band. When this compound is doped with divalent alkaline-earth cations $\mathrm{A}^{2+}(\mathrm{Sr}, \mathrm{Ba})$, a charge redistribution from $\mathrm{Mn}^{3+}$ to $\mathrm{Mn}^{4+}$ occurs. In a certain range of doping $(0.2 \div 0.5)$ these compounds show simultaneously ferromagnetism and metallic behaviour. This effect has been explained by Zenner in the double-exchange model [2]. According to this, the conduction is achieved through the hopping of an electron in the $e_{\mathrm{g}}$ state of the $\mathrm{Mn}^{3+}$ ions into the unoccupied $e_{\mathrm{g}}$ band of the $\mathrm{Mn}^{4+}$ ions. Later it has been shown

\footnotetext{
*corresponding author; e-mail: mdemeter@uos.de
} 
that the electron-phonon interaction also plays a very important role [3]. The microscopic origin of the strong electron-phonon coupling is the large Jahn-Teller effect which occurs for $d^{4+}$ ions in an octahedral environment [4].

The electronic and magnetic diagram of $\mathrm{La}_{1-x} \mathrm{Sr}_{x} \mathrm{MnO}_{3}$ has been established from electrical and magnetic measurements. Curie temperatures of $145 \mathrm{~K}, 309 \mathrm{~K}$, and $369 \mathrm{~K}$ for $x=0.1,0.2,0.3$ were also determined [5]. For $x<0.1$ the system is a canted AFM insulator and for $0.1<x<0.17$ it is FM insulator. For $x=0.175$ at room temperature there is also a structural transition from orthorhombic to rhombohedral. For $x>0.2$ a transition from FM insulator to FM metal takes place. The crystal structure of $\mathrm{La}_{1-x} \mathrm{Ba}_{x} \mathrm{MnO}_{3}$ changes from orthorhombic through rhombohedral to cubic $(x>0.35)$ with increasing concentration. The Curie temperatures of $\mathrm{La}_{1-x} \mathrm{Sr}_{x} \mathrm{MnO}_{3}$, where $x=0.1,0.2,0.3$ are $145 \mathrm{~K}, 309 \mathrm{~K}$, and $369 \mathrm{~K}$, respectively.

$\mathrm{X}$-ray photoelectron and X-ray emission spectroscopies (XPS, XES) are known as powerful tools to investigate the electronic structure of perovskites and the changes with carriers doping. In this paper we present new results of XPS and XES studies of the single crystals of $\mathrm{La}_{1-x} \mathrm{Sr}_{x} \mathrm{MnO}_{3}(x<0.3)$ and $\mathrm{La}_{0.8} \mathrm{Ba}_{0.2} \mathrm{MnO}_{3}$. Our experimental spectra are compared with the previous band structure calculations [6].

\section{Experimental}

Single crystals of $\mathrm{La}_{0.8} \mathrm{Ba}_{0.2} \mathrm{MnO}_{3},\left(\mathrm{La}_{0.9} \mathrm{Sr}_{0.1}\right)_{0.9} \mathrm{MnO}_{3}, \mathrm{La}_{0.8} \mathrm{Sr}_{0.2} \mathrm{MnO}_{3}$, and $\mathrm{La}_{0.7} \mathrm{Sr}_{0.3} \mathrm{MnO}_{3}$ were grown by the floating-zone method using the radiation heating of the zone by a xenon lamp at the Moscow Institute of Steel and Alloys by Ya.M. Mukovskii.

The X-ray photoelectron spectra were recorded with an ESCA spectrometer from Physical Electronics (PHI 5600 ci) using monochromatic $\mathrm{Al} K_{\alpha}$ radiation. All samples were measured after breaking in a high vacuum ( $10^{-9}$ torr). All spectra were recorded at room temperature. They were calibrated using an Au-foil $\left(E_{\mathrm{B}}\left(4 f_{7 / 2}\right)=84.0 \mathrm{eV}\right)$. The energy resolution as determined at the Fermi level of the Au-foil was $\sim 0.4 \mathrm{eV}$.

The $\mathrm{Mn} L_{\alpha}\left(3 d 4 s \rightarrow 2 p_{3 / 2}\right.$ transitions $)$ and $\mathrm{O} K_{\alpha}(2 p \rightarrow 1 s$ transitions $)$ $\mathrm{X}$-ray emission spectra were measured on the RSM-500-type X-ray vacuum spectrometer with a diffraction grating $(N=600$ lines $/ \mathrm{mm}, R=6 \mathrm{~m})$ and electron excitation. The spectra were recorded in the first order of reflection by a secondary electron multiplier with a CsI photocathode. The energy resolution was $0.4 \mathrm{eV}$. The X-ray tube was operated at $V=4.0 \mathrm{keV}$ and $0.25 \mathrm{~mA}$.

\section{Results and discussions}

In Fig. 1a the valence band spectra of $\mathrm{La}_{0.7} \mathrm{Sr}_{0.3} \mathrm{MnO}_{3}, \mathrm{La}_{0.8} \mathrm{Sr}_{0.2} \mathrm{MnO}_{3}$, and $\left(\mathrm{La}_{0.9} \mathrm{Sr}_{0.1}\right)_{0.9} \mathrm{MnO}_{3}$ are presented. They exhibit a structured wide band followed by a lower binding energy tail close to the Fermi level. The peaks at $6 \mathrm{eV}$ and $2.5-4 \mathrm{eV}$ correspond to the $\mathrm{O} 2 p$ states hybridized with $M n t_{2 \mathrm{~g}}$ states. The low 
intensity feature close to the Fermi level that is centred at $\sim 0.7 \mathrm{eV}$ is due to the emission from the $e_{\mathrm{g}}$ states. The most prominent peak at $6 \mathrm{eV}$ below the Fermi level does not change with $x$. The intensity of the La $5 p$ peak, located at $\sim 17 \mathrm{eV}$ decreases with $\mathrm{Sr}$ doping, whereas $\mathrm{Sr} 4 p_{3 / 2}$ peak at $\sim 19 \mathrm{eV}$ is increasing with $x$. The peak due to $O 2 s$ states is located at $21.5 \mathrm{eV}$. Similar studies on $\mathrm{La}_{0.84} \mathrm{Sr}_{0.16} \mathrm{MnO}_{3}$ single crystal [7] and polycrystalline samples $(x=0.0 \div 0.9)[8]$ were reported.
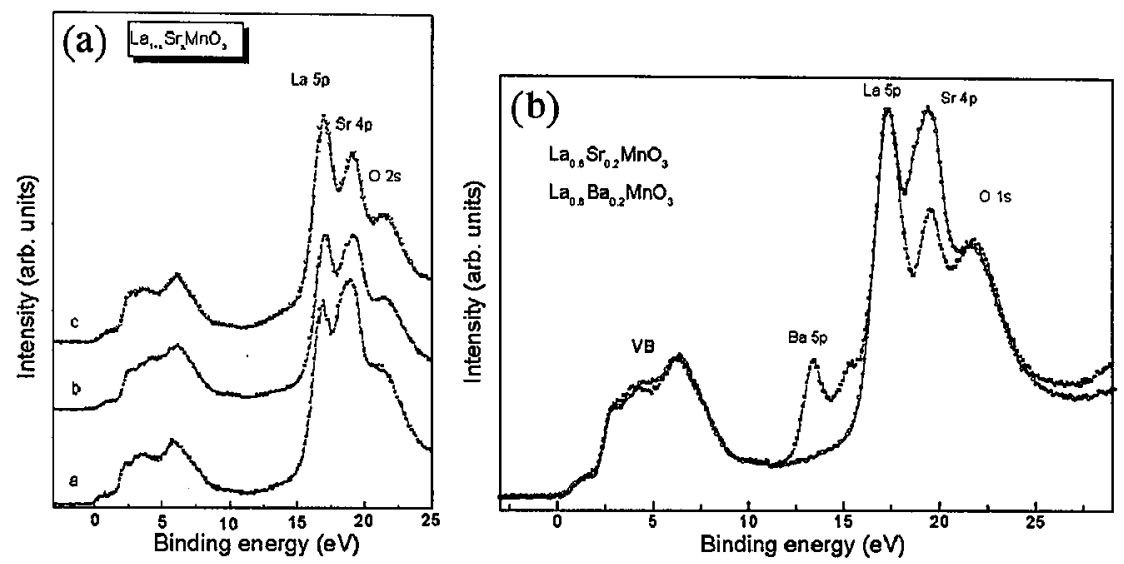

Fig. 1. (a) XPS valence band spectra of the single crystals $\mathrm{La}_{0.7} \mathrm{Sr}_{0.3} \mathrm{MnO}_{3}-a$, $\mathrm{La}_{0.8} \mathrm{Sr}_{0.2} \mathrm{MnO}_{3}-b$ and $\left(\mathrm{La}_{0.9} \mathrm{Sr}_{0.1}\right)_{0.9} \mathrm{MnO}_{3}-c$; the inset shows a comparison between $a$ - up triangle and $c$ - line from -2 to $10 \mathrm{eV}$. (b) Comparison of the XPS valence band spectra of $\mathrm{La}_{0.8} \mathrm{~A}_{0.2} \mathrm{MnO}_{3}(\mathrm{~A}=\mathrm{Sr}, \mathrm{Ba})$.

In Fig. 1b we present a comparison of the XPS valence band spectra of the single crystals $\mathrm{La}_{0.8}(\mathrm{Sr}, \mathrm{Ba})_{0.2} \mathrm{MnO}$. They exhibit similar features, a wide band and a low structure close to the Fermi level. There is a difference in the feature located at about $5 \mathrm{eV}$ where $\mathrm{O} 2 p$ contribution dominates. For Ba doped compound $(x=0.35)$ it was found that the feature corresponding to this energetic value is not enhanced at a photon energy of $52 \mathrm{eV}$ (the $\mathrm{Mn} 3 p$ resonance edge) indicating that it has a very little Mn $3 d$ character [9]. We assign the structure close to the Fermi level to $e_{\mathrm{g}}$ states, whereas the states at $2.9 \mathrm{eV}$ are assigned to $t_{2 \mathrm{~g}}$ states $[6,9]$. The next feature at $\sim 4 \mathrm{eV}$ is due to $\mathrm{O} 2 p$ states hybridised with Mn $t_{2 \mathrm{~g}}$ states. The highest peak at $\sim 6 \mathrm{eV}$ is the same as in the similar Sr-doped compound. Linear muffin-tin orbital (LMTO) band structure calculations performed on the $\mathrm{La}_{1-x} \mathrm{Ba}_{x} \mathrm{MnO}$ system [10] have shown that for $x=0.2$ the contribution to the total density of states (DOS) at the Fermi level comes mainly from $e_{\mathrm{g}}$ states, whereas for $x=0.4$, in the half-metallic regime $(x>0.33)$ the density of states at $E_{\mathrm{F}}$ is due solely to $e_{\mathrm{g}}$ electrons. In the Ba-doped sample, the peak located at $13.4 \mathrm{eV}$ is due to $\mathrm{Ba} 5 p$ states. The feature at binding energies higher than $25 \mathrm{eV}$ in the $\mathrm{Ba}$-doped sample is given by the $\mathrm{Ba} 5 s$ peak contribution (not shown here).

In Fig. 2 XPS valence band together with XES Mn $L_{\alpha}$ and $\mathrm{O} K_{\alpha}$ spectra are shown for $\mathrm{LaMnO}_{3}$ and $\mathrm{Sr}(\mathrm{Ba})$-doped samples $(x=0.2)$. Mn $L_{\alpha} \mathrm{X}$-ray emission 
arises from the $3 d 4 s \rightarrow 2 p_{3 / 2}$ electronic transition and reflects the Mn $3 d$ distribution in the valence band. O $K_{\alpha} \mathrm{X}$-ray emission corresponds to the $2 p \rightarrow 1 s$ transition and reveals the $\mathrm{O} 2 p$ partial density of states. The XES Mn $L_{\alpha}$ and O $K_{\alpha}$ spectra were brought to the binding energy scale by using the Mn $2 p_{3 / 2}$ and $\mathrm{O} 1 s$ binding energies, respectively. The $\mathrm{Mn} L_{\alpha}$ and $\mathrm{O} K_{\alpha}$ XES spectra are localized in the same energy area, which indicates a strong $\mathrm{Mn} 3 d-\mathrm{O} 2 p$ hybridization. In the $\mathrm{O} K_{\alpha}$ spectrum, the maximum at $\sim 3.5 \mathrm{eV}$ corresponds to the antibonding $p d \pi$ states and the shoulder at $6 \mathrm{eV}$ reflects the bonding $p d \sigma$ states. Our experimental results are in good agreement with local spin-density approximation (LSDA) calculations [6].

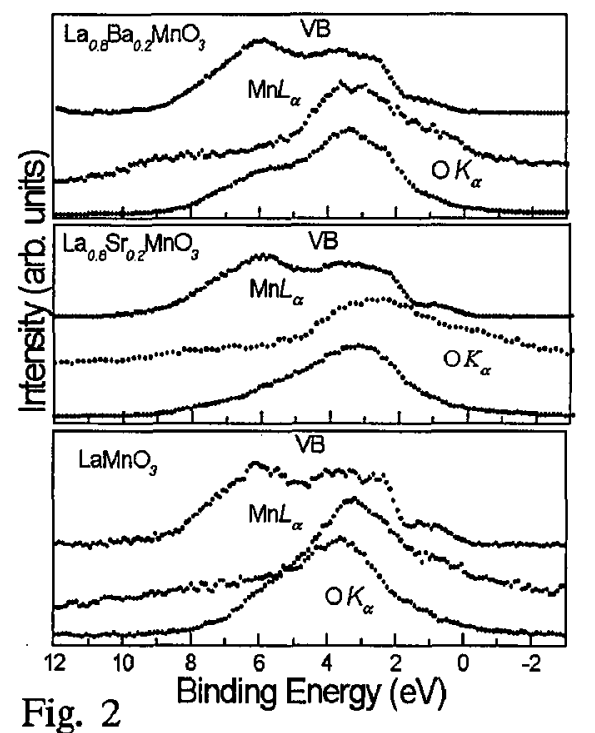

Fig. 2

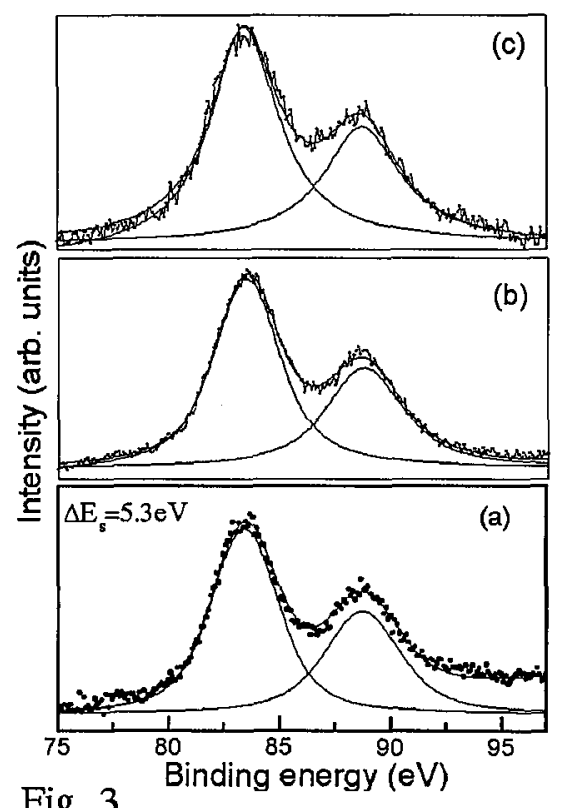

Fig. 3

Fig. 2. XPS and XES spectra of $\mathrm{LaMnO}_{3}, \mathrm{La}_{0.8} \mathrm{Sr}_{0.2} \mathrm{MnO}_{3}$, and $\mathrm{La}_{0.8} \mathrm{Ba}_{0.2} \mathrm{MnO}_{3}$.

Fig. 3. XPS Mn 3s splitting of the single crystals of $\mathrm{La}_{0.7} \mathrm{Sr}_{0.3} \mathrm{MnO}_{3}$ (a), $\mathrm{La}_{0.8} \mathrm{Sr}_{0.2} \mathrm{MnO}_{3}$ (b), and ( $\left.\mathrm{La}_{0.9} \mathrm{Sr}_{0.1}\right)_{0.9} \mathrm{MnO}_{3}$ (c).

The $3 s$ exchange splitting in transition metal compounds is due to the exchange interaction between the $3 s$ core hole created in a XPS process and $3 d$ electrons. The magnitude of the $\mathrm{Mn} 3 s$ splitting $\left(\Delta E_{s}\right)$ depends on the valence state of the Mn ions. In Fig. 3 the Mn $3 s$ XPS spectra of $\left(\mathrm{La}_{0.9} \mathrm{Sr}_{0.1}\right)_{0.9} \mathrm{MnO}_{3}$, $\mathrm{La}_{0.8} \mathrm{Sr}_{0.2} \mathrm{MnO}_{3}$, and $\mathrm{La}_{0.7} \mathrm{Sr}_{0.3} \mathrm{MnO}_{3}$ single crystals are presented. The highest peak is located at $\sim 83.5 \mathrm{eV}$. In Ref. [8] it has been reported that the Mn $3 s$ splitting of polycrystalline $\mathrm{La}_{1-x} \mathrm{Sr}_{x} \mathrm{MnO}_{3}(x=0.0 \div 0.9)$ monotonically decreases with $x$. However, our spectra on single crystals samples show that the value of the Mn $3 s$ splitting (5.3 eV) does not change with Sr doping from $x=0.0$ to $x=0.3$. This indicates that the doped holes have mainly oxygen $p$ character. The large value of the splitting is consistent with the high-spin configurations. 


\section{Conclusions}

We have presented new XPS and XES results on manganese doped perovskites, single crystals. The same $3 s$ splitting of the Sr-doped samples with $x<0.3$ indicates the oxygen $2 p$ character of the doped holes. The large value of the splitting shows that the Mn $3 d$ electrons are in the high spin state. The Mn $L_{\alpha}$ and $O K_{\alpha}$ XES spectra are localised in the same energy area indicating strong Mn $3 d-\mathrm{O} 2 p$ hybridisation. Our data are in good agreement with the previous LSDA results.

\section{Acknowledgments}

The financial support from DFG, especially Graduiertenkolleg is gratefully acknowledged. One of the authors (V.R. G.) want to thank for the financial support from the RFBR-99-02-16280 Project. We thank Ya.M. Mukovskii for supplying the single crystals.

\section{References}

[1] A.P. Ramirez, J. Phys., Condens. Matter 9, 8171 (1997); J.M.D. Coey, M. Viret, S. von Molnár, Adv. Phys. 48, 167 (1999).

[2] C. Zener, Phys. Rev. 82, 403 (1951); J. Goodenough, Phys. Rev. 100, 564 (1955).

[3] A.J. Millis, P.B. Littlewood, B.I. Shraiman, Phys. Rev. Lett. 74, 5144 (1995); A.J. Millis, B.I. Shraiman, R. Mueller, Phys. Rev. Lett. 77, 175 (1996).

[4] J.R. Fletcher, K.W.H. Stephens, J. Phys. C, Solid State Phys. 2, 444 (1969).

[5] Y. Tokura, A. Urushibara, Y. Moritomo, T. Arima, A. Asamitsu, G. Kido, N. Furukuwa, J. Phys. Soc. Jpn. 63, 3931 (1994); A. Urushibara, Y. Moritomo, T. Arima, A. Asamitsu, G. Kido, Y. Tokura, Phys. Rev. B 51, 14103 (1995).

[6] S. Sapathy, Z.S. Popović, F.R. Vukajlović, Phys. Rev. Lett. 76, 960 (1996); D.D. Sarma, N. Shanthi, S.R. Barman, N. Hamada, H. Sawada, T. Terakura, Phys. Rev. Lett. 75, 1126 (1995).

[7] Y. Kuwata, S. Suga, S. Imada, A. Sekiyama, S. Ueda, T. Iwasaki, H. Harada, T. Muro, T. Fukawa, K. Ashida, H. Yoshioka, T. Terauchi, J. Sameshima, H. Kuwahara, Y. Maritomo, Y. Tokura, J. Electron Spectrosc. Relat. Phenom. 88-91, 281 (1998).

[8] T. Saitoh, A.E. Bocquet, T. Mizokawa, H. Namatame, A. Fujimori, M. Abate, Y. Takeda, M. Takano, Phys. Rev. B 51, 13942 (1995).

[9] D.N. Mcllroy, C. Waldfried, Jiandi Zhang, J.W. Choi, F. Foong, S.H. Liou, P.A. Dowben, Phys. Rev. B 54, 17438 (1996).

[10] S.J. Youn, B.I. Min, Phys. Rev. B 56, 12046 (1997). 